\title{
LETTERS
}

\section{Strong administrative support needed to improve wait times for hip fracture}

I would like to praise Pincus and colleagues for their article on wait times for urgent hip fracture surgery ${ }^{1}$ and commend them for their ongoing work. As a community-based orthopedic surgeon who works in a medium-sized hospital servicing rural and very rural areas, I believe there is an area identified in the article that merits further review in the scope of rural practice - that hospitallevel delays seem to be at the root of much variation in wait times.

The authors suggest that hip fracture care needs to be streamlined and have clear algorithms, such as the one developed in Manitoba. ${ }^{1,2}$ This initiative focused on expediting transfers, cutting down on unnecessary preoperative tests and steps, increasing the availability of operating room time for hip fractures, and collaboration with referral centres to repatriate patients postoperatively. Although this model is efficacious and is likely to be embraced by surgeons and patients alike, without strong administrative support, it remains an unachievable aspiration.

At my hospital, our current benchmark for urgent hip fracture surgery is set at 48 hours from time of admission to time of surgery, but this is likely and justifiably going to be shortened to 24 hours, and perhaps even less time. In a recent CMAJ article, Sobolev and colleagues lean this way in suggesting that hip fracture surgeries should be done on the day of admission or the day thereafter. ${ }^{3}$ The work required to achieve this target for medium- and small-sized community hospitals is likely to be tremendous.

Sheehan and colleagues provide further insight into the effect of the treatment setting on hip fracture surgery, suggesting that medium community hospitals fare the worst in terms of postsurgical death, and medium and small community hospitals also fare poorly on the risk of in-hospital death after hip fracture. ${ }^{4}$ Sheehan and colleagues recognize their inability to adjust for urban, rural or remote location as a limitation of their study. I think we need to look very critically at our small- and medium-sized community hospitals, especially those in rural areas, and the orthopedic surgical care given therein, as I fear that funding and resources are not keeping pace with heightened standards of care. As such, the strain being heaped on the system may be approaching unsustainable levels at some of these centres. I, for one, can attest that it is being felt acutely.

Where I work, my colleagues and I can access the operating room for after-hours work (evenings, nights and weekends) only if life or limb is imminently threatened, which is not the case with hip fractures. We have access to 9.45 hours of operating room time weekly, split over 3 days, to do acute fracture care, but the time is available in defined blocks. If a hip fracture surgery cannot meet the 48-hour benchmark within these 3 slots, we are expected to cancel elective cases to facilitate the surgery but are not given extra time to rebook those patients. We are reminded of the goal to reach benchmarks, but the responsibility to make this happen is left with the surgeon, while the system does little to support this goal. My colleagues in similar-sized hospitals across the country echo these sentiments.

In their article, Pincus and colleagues suggest that surgeons are trying their best to maintain standard of care, but that system-level factors are often what hold them back. My postulation is that this is probably even more true in rural and very rural areas.

I am a rural orthopedic surgeon, but I grew up in a very rural area - in a town of 100 people in coastal Newfoundland and Labrador, where transfer to the nearest small hospital is $100 \mathrm{~km}$, and transfer to the medium community hospital where I now work (and the nearest orthopedic care) is $200 \mathrm{~km}$. These roads in winter are, at times, completely impassable. People who live in these areas accept the fact that their emergency care is not held to the same standard as that of their lessrural and urban counterparts. Although some of the factors that make this true cannot be changed (blizzards cannot be halted), some of them can.

We can do better with hip fracture care - in timeliness, in postoperative rehabilitation and in ensuring that there is equity with regard to allied health services across the spectrum of hospital settings - but we cannot do this without strong administrative support. I believe it is our duty as physicians to raise awareness and bring our administrators on board to enact system-wide collaboration and change. In a world of increasing negativity, we have to be the leaders to ignite positive change where it can be made.

\section{Tracey M. Wentzell MDCM/MBA}

Orthopedic surgeon, Western Memorial Regional Hospital, Corner Brook, NL

Cite as: CMAJ 2019 May 21;191:E566. doi: $10.1503 / \mathrm{cmaj} .71978$

\section{References}

1. Pincus D, Wasserstein D, Ravi B, et al. Reporting and evaluating wait times for urgent hip fracture surgery in Ontario, Canada. CMAJ 2018;190: E702-9.

2. Bohm E, Loucks L, Wittmeier K, et al. Reduced time to surgery improves mortality and length of stay following hip fracture: results from an intervention study in a Canadian health authority. Can J Surg 2015;58:257-63.

3. Sobolev B, Guy P, Sheehan KJ, et al.; for the Canadian Collaborative Study of Hip Fractures. Mortality effects of timing alternatives for hip fracture surgery. CMAJ 2018;190:E923-32.

4. Sheehan KJ, Sobolev B, Guy P, et al. In-hospital mortality after hip fracture by treatment setting. CMAJ 2016;188:1219-25.

Competing interests: None declared. 